\title{
Brazelton Neonatal Behavioral Assessment Scale: A psychometric study in a Portuguese sample
}

\section{Raquel Costa*, Bárbara Figueiredo, Iva Tendais, Ana Conde, Alexandra Pacheco, César Teixeira}

Department of Psychology, University of Minho, Campus de Gualtar, 4700-320 Braga, Portugal

\section{A R T I C L E I N F O}

\section{Article history:}

Received 1 June 2009

Received in revised form

21 December 2009

Accepted 18 July 2010

\section{Keywords:}

Neonatal Behavioral Assessment Scale

Psychometric properties

Principal component analysis

Reliability

Neurobehavior organization

\begin{abstract}
A B S T R A C T
Background: The Neonatal Behavioral Assessment Scale (NBAS, Brazelton \& Nugent, 1995) is an instrument conceived to observe the neonatal neurobehavior. Data analysis is usually performed by organizing items into groups. The most widely used data reduction for the NBAS was developed by Lester, Als, and Brazelton (1982).

Objective: Examine the psychometric properties of the NBAS items in a sample of 213 Portuguese infants.

Method: The NBAS was performed in the first week of infant life ( 3 days \pm 2 ) and in the seventh week of life (52 days \pm 5 ).

Results: Principal component analyses yielded a solution of four components explaining $55.13 \%$ of total variance. Construct validity was supported by better neurobehavioral performance of 7-week-old infants compared with 1-week-old infants.

Conclusion: Changes in the NBAS structure for the Portuguese sample are suggested compared to Lester factors in order to reach better internal consistency of the scale.
\end{abstract}

(c) 2010 Elsevier Inc. All rights reserved.

\section{Introduction}

The Neonatal Behavioral Assessment Scale (NBAS) was conceived with the purpose of assessing the contribution of the neonate to the parent-infant system (Brazelton \& Nugent, 1995), assuming that the newborn is both competent and organized (Brazelton, 1973). The main aim is to gather a comprehensive profile of neonatal functioning by describing the full range of neonatal behavior, including competencies and strengths, as well as difficulties or deviation. This instrument has proved to be of great clinical interest because it detects infants at risk for later developmental problems. Additionally, it is also used in clinical practice as an intervention instrument for improving mother-infant bonding in risk samples (Brazelton \& Nugent, 1995). The study of this instrument in a sample of Portuguese infants is relevant in order to document cultural variation in neonatal behavior. The NBAS is composed of 28 behavioral items scored on a 9 point-scale, 14 reflex items scored on a 4 point-scale, and 7 supplementary items scored on a 9 point-scale. Data analyses can be difficult because of the high number of correlated behavioral items, so the items need to be organized in groups in order to reduce the probability of chance findings from multiple analyses. This can be done through the construction of a priori factors or factor analytical studies. One of the first attempts to reduce the NBAS behavioral items using factor analysis was developed by Osofsky and O'Connell (1977) in a sample of 328 infants. They found a six factor solution: (1) responsivity, (2) reactivity, (3) habituation, (4) state control and the fifth and sixth factors were named motor maturity. Als (1978) conceived a procedure to reduce

\footnotetext{
* Corresponding author. Tel.: +351253 604241; fax: +351253678987.

E-mail addresses: rcosta@iep.uminho.pt, rqlcosta@gmail.com (R. Costa).
} 
the NBAS behavioral item scores to four dimensions based on postulated neurophysiological and psychological processes in the neonate: (1) interactive processes; (2) motoric processes; (3) organization processes: state control; (4) organizational processes: physiological response to stress. Kaye (1978) reduced the behavioral item scores to a few dimensions using and comparing four different approaches: (1) factor analysis, (2) canonical correlation, (3) lumping and smoothing, and (4) multiple regression. Strauss and Rourke (1978) performed a factorial analysis and came up with an eight factor solution for the behavioral items. Sostek, Davitt, Renzi, Born, and Kiely (1982) performed a factor study with pre-term infants that revealed a four factor structure: (1) alertness; (2) quieting; (3) habituation; (4) irritability and (5) motor functioning. The aforementioned studies yielded quite different results which might be due to different statistical procedures and sample characteristics.

The most widely used data reduction system for the NBAS items was developed by Lester, Als, and Brazelton (1982) that conceived a seven factor scoring based on both previous factorial analysis studies and their own statistics. The factors are: (1) habituation, (2) orientation, (3) motor, (4) range of state, (5) regulation of state, (6) autonomic stability and (7) reflexes. The habituation factor refers to the ability to respond to and inhibit discrete stimuli while asleep. The orientation factor includes the ability to attend to visual and auditory stimuli and the quality of overall alertness. The motor factor measures motor performance and the quality of movement and tone. The range of state is a measure of infant arousal and state lability. The regulation of state reports the infant's ability to regulate his/her state in the face of increasing levels of stimulation. The autonomic stability records signs of stress related to homeostatic adjustments of the central nervous system, whereas the reflexes are a record of the number of abnormal reflexes (Brazelton \& Nugent, 1995).

Several studies have been conducted using NBAS Lester factors: studies of neuropsychological development (e.g. Field, Diego, Hernandez-Reif, Schanberg, \& Kuhn, 2002), risk factors (e.g. Ohgi et al., 2003), effects of maternal substance use (Myers et al., 2003), inter-cultural studies (Loo, Ohgi, Zhu, Howard, \& Chen, 2005), gender differences (Boatella-Costa, Moragas, Mussons, Deu, \& Zurita, 2007) and intervention studies (Ohgi, Fukuda, Akiyama, \& Gima, 2004). Considering the importance of assessing the psychometric properties of these factors, two lines of studies have emerged using: (1) confirmatory factorial analyses and (2) exploratory factorial analyses with comparison of the psychometric properties of the derived factor with the psychometric properties of the Lester et al. (1982) factors. To date, only two confirmatory factor analyses were reported to evaluate the psychometric properties of the Lester factors, one with premature infants and the other in a normative sample. Azuma, Malee, Kavanagh, and Deddish (1991) performed confirmatory factor analyses to analyze the psychometric properties of Lester's factors and compare them to three other data reduction models in a sample of 166 pre-term infants. None of the four models could be confirmed, the maximum likelihood factor analysis failed to confirm Lester's factors system or any of the three alternative models. The authors conclude that the lack of empirical support for the Lester model could be due to the specificities of the premature sample behavior. McCollam, Embretson, Mitchell, and Horowitz (1997) assessed the fit of the NBAS data in a sample of 160 healthy infants to variation of the Lester factor model. Four different models of confirmatory factor analysis were used and compared indicating that the general configuration of the Lester factors is a good baseline for reducing the NBAS items, although some modifications are suggested.

Two exploratory factor analyses have been reported to reduce the NBAS items with posterior comparison of the derived factors psychometric properties with the psychometric properties of Lester's factors. Jacobson, Fein, Jacobson, and Schwartz (1984) examined the properties of the Lester's behavioral factors in a sample of 160 neonates and compared them with a revised set of factors. They found that the revised factors exhibited greater internal consistency, stronger test-retest reliability and orthogonality compared with the Lester factors. More recently, Moragas, Deu, Mussons, Boatella Costa, and Zurita (2007), in a psychometric evaluation of the scale with a sample of 220 infants, used exploratory factor analysis and obtained five factors identical to the Lester factors. The reliability analysis revealed moderate to high scores.

The aim of this study is to reduce the NBAS behavioral items into factors using principal component analyses. It is also our aim to compare the reliability of the extracted factors in our sample of 213 Portuguese infants with the reliability of the Lester factors.

\section{Method}

\subsection{Participants}

The sample was composed of 213 Portuguese infants ( $54.7 \%$ males) born of vaginal delivery (44.4\%) and cesarian section (55.6\%). Most newborns were full-term (94.1\%) and had no need for reanimation at birth (92.9\%). The apgar score in the 1st and 5 th minute was equal or above 7 in most cases (90\% and 97.7\%). The weight ranged from 1830 to $4430 \mathrm{~g}(M=3132.7 \mathrm{~g}$, $\mathrm{SD}=480.8)$ and was equal or above $2500 \mathrm{~g}$ in almost all sample $(93.4 \%)$. The height ranged from $42 \mathrm{to} 54 \mathrm{~cm}(M=48.4 \mathrm{~cm}$, $\mathrm{SD}=2.3)$ and the cephalic perimeter ranged from 30 to $45.5 \mathrm{~cm}(M=35.1 \mathrm{~cm}, \mathrm{SD}=6.1)$.

\subsection{Procedure}

The parents of the infants were contacted during pregnancy in three public health care institutions from Porto district (Portugal). Informed consent was given prior to birth. Infants neurobehavioral functioning was evaluated using the Neonatal Behavioral Assessment Scale (NBAS, Brazelton \& Nugent, 1995). Half of the sample was examined in the first week of life ( 3 days \pm 2 ), while the other half was examined in the seventh week of life ( 52 days \pm 5 ). This examination was conducted 
Table 1

Inter-item correlation and alpha of Cronbach for the Lester habituation and orientation factor in our sample.

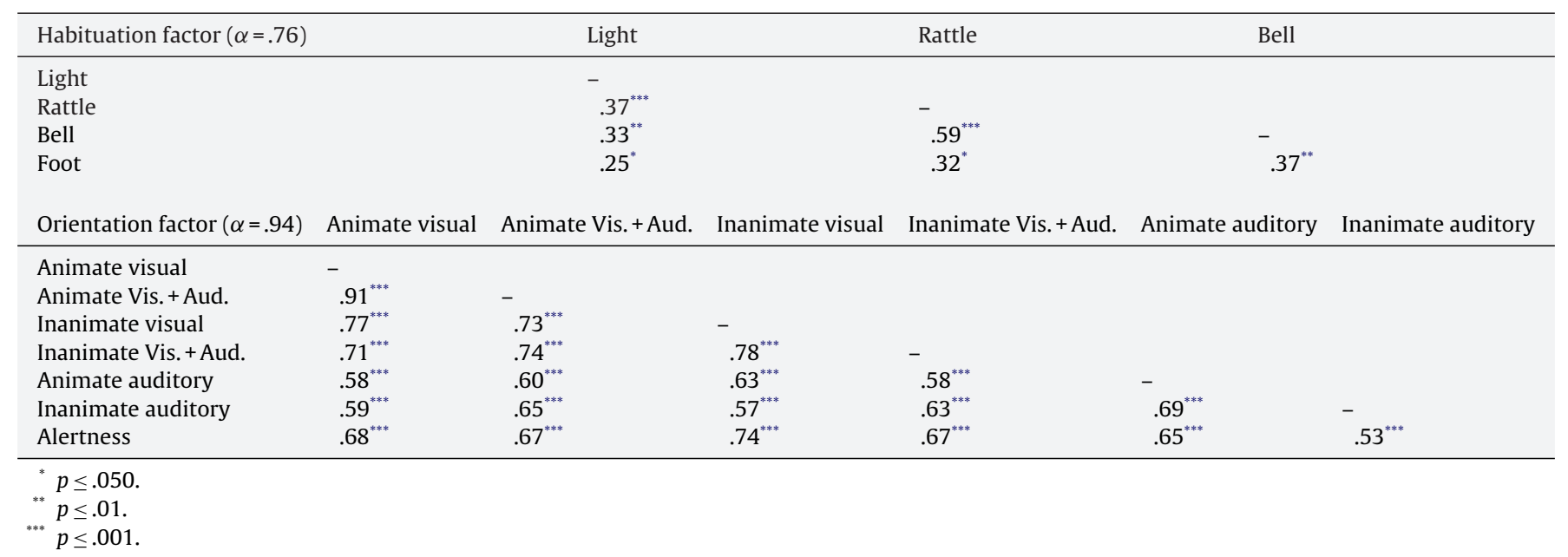

in a particular sequence according to author's instructions (Brazelton \& Nugent, 1995) midway between feedings in a quiet and semi-darkened room with a temperature of $22-27^{\circ} \mathrm{C}$. The NBAS was scored immediately after performed by a trained, certified and independent reliable examiner.

Because the administration of some items required the infant to be in specific states to be administered (e.g., the habituation package should be administered preferable in the state 1 (deep sleep) or 2 (light sleep) and the orientation package must be administered while the infant is in an alert state (Brazelton \& Nugent, 1995), some could not be completed. The Lester orientation items are missing in 37 infants and the habituation items were scored for only 98 infants. Consolability was not scored on 27 infants that did not reach state 6 (crying) during the examination and self-quieting is missing on 23 infants that did not reach state 5 (alert, eyes open and considerable motor activity).

\subsection{Statistical analyses}

Principal component analyses with orthogonal rotation (varimax) were performed in order to reduce data into a more manageable size while maintaining as much of the original information as possible (Field, 2005). The items with midrange optimal scores were not recoded, since the recoded versions of these items were so weakly interrelated in previous studies (e.g. Jacobson et al., 1984; McCollam, Embretson, Horowitz, \& Mitchell, 1996). Data in the first analysis were conducted using Kaiser's criterion of retaining all factors with eigenvalues over 1 (Kaiser, 1960, 1974). Data in the following analysis were forced into a 4-component solution.

For the NBAS total score, behavioral and reflexes items were recoded so that a better performance corresponds to higher punctuation and were then summed. Internal consistency was assessed since the principal component analysis must be accompanied by the internal consistency of the factors. It was measured through item-total correlation, average interitem correlation and Cronbach's alpha coefficients. Nunnally and Bernstein (1994) suggest that good internal consistency is indicated when Cronbach's alpha is over .70 and mean inter-item correlations exceed .15. Field (2005) also argues that item-total correlation should be over 30 .

The Cronbach's alpha coefficient of extracted factors was then compared with the Cronbach's alpha coefficient of the Lester et al. (1982) factors in our sample.

Descriptive statistics of the factor items was performed as well as correlation analyses (Pearson correlation) between the NBAS factors and factors-total.

Construct validity was also evaluated using MANOVAs to identify differences in the NBAS factor scores and t-test to identify differences in the NBAS total score between 1-week-old infants and 7-week-old infants.

\section{Results}

\subsection{Principal component analysis and internal consistency}

The principal components analyses were performed with sixteen NBAS items. The four habituation items and the seven orientation items were not included in this analysis because there is a consistent theoretical background that supports both factors. Additionally, empirical data shows that the items within these factors are highly correlated in our sample (see Table 1) as in other samples (e.g., Jacobson et al., 1984; Moragas et al., 2007; Osofsky \& O'Connell, 1977). Furthermore the high rate of missing data in our sample would compromise the statistical analysis. Nonetheless, these factors show good internal consistency in our sample and in other studies (e.g., Jacobson et al., 1984; Moragas et al., 2007). 
Table 2

Principal component analysis with varimax rotation (Kaiser criterion of retaining all factors with eigenvalues over 1 ).

\begin{tabular}{|c|c|c|c|c|c|c|c|}
\hline & \multicolumn{5}{|c|}{ Component } & \multirow[t]{2}{*}{ Comm. } & \multirow[t]{2}{*}{ ITC correlation } \\
\hline & 1 & 2 & 3 & 4 & 5 & & \\
\hline \multicolumn{8}{|l|}{ Regulation of state } \\
\hline Self-quieting & 0.84 & -0.21 & 0.11 & -0.03 & -0.01 & .76 & .70 \\
\hline Consolability & 0.68 & -0.29 & 0.03 & -0.02 & 0.18 & .58 & .50 \\
\hline Hand-to-mouth & 0.61 & 0.22 & 0.18 & 0.19 & -0.41 & .66 & .38 \\
\hline Pull-to-sit & -0.44 & -0.20 & -0.36 & 0.38 & -0.35 & .63 & .40 \\
\hline \multicolumn{8}{|l|}{ Range of state } \\
\hline Irritability & 0.08 & 0.82 & 0.11 & 0.08 & 0.22 & .74 & .63 \\
\hline Lability of states & -0.03 & 0.81 & 0.07 & -0.06 & -0.14 & .69 & .63 \\
\hline Rapidity of build-up & -0.35 & 0.61 & 0.05 & -0.03 & 0.06 & .50 & .53 \\
\hline Peak of excitement & -0.37 & 0.51 & -0.16 & 0.38 & -0.01 & .58 & .39 \\
\hline \multicolumn{8}{|l|}{ Autonomic stability } \\
\hline Startles & 0.10 & -0.08 & 0.81 & 0.09 & -0.15 & .70 & .55 \\
\hline Motor maturity & -0.11 & -0.10 & -0.66 & 0.24 & -0.23 & .56 & .42 \\
\hline \multicolumn{8}{|l|}{ Motor } \\
\hline Defensive & 0.00 & -0.04 & 0.05 & 0.79 & 0.00 & .63 & .36 \\
\hline Activity level & -0.15 & 0.05 & 0.41 & 0.64 & 0.09 & .61 & .34 \\
\hline Cuddliness & 0.49 & 0.17 & -0.13 & 0.55 & -0.07 & .59 & .23 \\
\hline \multicolumn{8}{|l|}{ Component 5} \\
\hline Lability of skin color & 0.05 & 0.08 & 0.04 & 0.05 & 0.91 & .84 & \\
\hline Eigenvalues & 2.46 & 2.26 & 2.05 & 1.88 & 1.31 & & \\
\hline \% Variance explained & 15.39 & 14.09 & 12.83 & 11.77 & 8.18 & & \\
\hline$\%$ Cumulative variance & 15.39 & 29.47 & 42.31 & 54.07 & 62.25 & & \\
\hline
\end{tabular}

The Bartlett's test of sphericity shows values of $X_{(120)}^{2}=532.588 ; p<.001$, indicating that there are significant correlations and data is adequate for principal component analysis. The Kaiser-Meyer-Olkin for measuring sample adequacy - KMO = .66 - is over the minimum of .50 recommended by Kaiser (1974).

The first principal component analysis generated five components with eigenvalues over 1 , accounting for $62.25 \%$ of the total variance. Table 2 shows the factor loadings, communalities, and item-total correlations.

This solution presents a fifth component with only one item. To overcome this issue, a principal component analysis was performed with data forced into a 4-component solution. This solution also allows an approach to the number of Lester's factors. Four components accounting for 55.13\% of the total variance were obtained. Table 3 shows the extracted components, factor loadings, communalities, average inter-item correlation, item-to-total correlation, Cronbach's alpha and Cronbach's alpha if item deleted. Five items demonstrated low item-to-total correlations (<.30): "defensive" (.28), "general tone" (.29), "lability of skin color" (.29), "cuddliness" (.29) and "activity level" (.25). We decided not to exclude the "defensive", "general tone" and "lability of skin color" items because the omission of these items would not increase the Cronbach's Alpha of the motor component, and none of these items load in other components. The item "cuddliness" was not excluded because although the omission would slightly increase the regulation of state component reliability, the item loads strongly on this component and the communality is over .50. Furthermore the average item correlation exceeds .15 (Nunnally \& Bernstein, 1994). Finally, the "activity level" item was also not excluded because, although the exclusion would increase the internal consistency of the autonomic stability component, this increment is not dramatic. Moreover the item communality is above .50 , the item loads strongly on this component, the average item correlation is over .50 and the omission of this item would leave the component with only two items.

Comparing the extracted component structure with Lester factors (see Table 4), we find that the extracted regulation of state and range of state components are composed of exactly the same items as the Lester factors. The extracted motor component is composed of four of the items in the Lester motor factor (pull-to-sit, motor maturity, defensive and general tone) plus the "lability of skin color" item. The extracted autonomic stability is composed of "startles" and "tremulousness" as in Lester factor plus the "activity level" item.

The Cronbach's alpha of the extracted components ranged from .54 to .74 (.74 - range of state, .60 - motor, .68 - regulation of state, and .54 - autonomic stability) (see Table 4). The Cronbach's alpha of the extracted range of state and autonomic stability components are higher than the Cronbach's alpha of the Lester range of state and autonomic stability factors in our sample. The Cronbach's alpha of the extracted motor component is similar to the Cronbach's alpha of the Lester motor factor in our sample. The extracted regulation of state component is composed of the exactly same items as the Lester regulation of state factor and so the Cronbach's alpha value is equal.

The descriptive statistics of the items are shown in Table 4. In the range of state component, the item with median score closest to optimal score is "peak of excitement", while the item with more variability is "rapidity of build-up". In the motor 
Table 3

Internal consistency and principal component analysis with varimax rotation (4 factor solution).

\begin{tabular}{|c|c|c|c|c|c|c|c|c|}
\hline & \multicolumn{4}{|c|}{ Component } & \multirow[t]{2}{*}{ Comm. } & \multirow[t]{2}{*}{ AIC } & \multirow[t]{2}{*}{ ITC } & \multirow{2}{*}{$\begin{array}{l}\alpha \text { if item } \\
\text { deleted }\end{array}$} \\
\hline & 1 & 2 & 3 & 4 & & & & \\
\hline Range state $(\alpha=.74)$ & & & & & & .419 & & \\
\hline Irritability & 0.80 & -0.22 & 0.16 & 0.09 & 0.72 & & 0.63 & .63 \\
\hline Lability of states & 0.78 & -0.10 & 0.11 & -0.02 & 0.63 & & 0.63 & .64 \\
\hline Rapidity of build-up & 0.66 & -0.01 & -0.25 & 0.03 & 0.50 & & 0.53 & .70 \\
\hline Peak of excitement & 0.61 & 0.41 & -0.13 & 0.10 & 0.57 & & 0.39 & .76 \\
\hline Motor $(\alpha=.60)$ & & & & & & .231 & & \\
\hline Pull-to-sit & -0.09 & 0.76 & -0.20 & -0.03 & 0.62 & & 0.55 & .41 \\
\hline Motor maturity & -0.05 & 0.63 & 0.06 & -0.39 & 0.56 & & 0.45 & .49 \\
\hline Defensive & 0.04 & 0.50 & 0.24 & 0.49 & 0.55 & & 0.28 & .59 \\
\hline General tone & 0.14 & 0.47 & -0.23 & 0.04 & 0.30 & & 0.29 & .59 \\
\hline Lability of skin color & 0.14 & -0.39 & -0.15 & 0.09 & 0.20 & & 0.29 & .58 \\
\hline Regulation state $(\alpha=.68)$ & & & & & & .345 & & \\
\hline Hand-to-mouth & 0.09 & -0.04 & 0.73 & 0.16 & 0.57 & & 0.47 & .61 \\
\hline Self-quieting & -0.36 & -0.35 & 0.71 & -0.01 & 0.76 & & 0.65 & .46 \\
\hline Cuddliness & 0.13 & 0.27 & 0.67 & 0.14 & 0.55 & & 0.29 & .71 \\
\hline Consolability & -0.40 & -0.31 & 0.52 & -0.04 & 0.53 & & 0.47 & .61 \\
\hline Autonomic stability $(\alpha=.54)$ & & & & & & .280 & & \\
\hline Activity level & 0.13 & 0.24 & 0.02 & 0.72 & 0.58 & & 0.25 & .60 \\
\hline Tremulousness & 0.12 & -0.27 & 0.12 & 0.70 & 0.60 & & 0.49 & .24 \\
\hline Startles & -0.13 & -0.31 & 0.08 & 0.70 & 0.60 & & 0.45 & .30 \\
\hline Eigenvalues & 2.46 & 2.33 & 2.07 & 1.96 & & & & \\
\hline \% Variance explained & 15.40 & 14.53 & 12.92 & 12.28 & & & & \\
\hline \% Cumulative variance & 15.40 & 29.94 & 42.86 & 55.13 & & & & \\
\hline
\end{tabular}

Note: Comm. = communalities; AIC = average item correlation; ITC=item-to-total correlation.

component, the item with median score closest to optimal score is "motor maturity" and the item with more variability is "pull-to-sit". The item of the regulation of state component with better median score is "cuddliness" whereas the selfquieting has more variability. Finally, in the autonomic stability component, the item tremulousness is the one with both median score closest to optimal score and more variability.

Table 4

Factor structure and internal consistency of the extracted factors and of Lester factors in our sample. Descriptive statistics of the items composing the extracted factors.

\begin{tabular}{|c|c|c|}
\hline Lester & Extracted & Mean (SD) \\
\hline Range of state $(\alpha=.50)$ & Range of state $(\alpha=.74)$ & \\
\hline Irritability $^{\mathrm{a}}$ & Irritability & $3.26(1.97)$ \\
\hline Lability states $^{\mathrm{a}}$ & Lability states & $3.08(1.65)$ \\
\hline Rapidity of build-up ${ }^{\mathrm{a}}$ & Rapidity of build-up & $3.81(2.19)$ \\
\hline Peak of excitement ${ }^{\mathrm{a}}$ & Peak of excitement & $5.11(1.63)$ \\
\hline Motor $(\alpha=.61)$ & Motor $(\alpha=.60)$ & \\
\hline Pull-to-sit & Pull-to-sit & $5.97(1.98)$ \\
\hline Motor maturity & Motor maturity & $6.79(1.64)$ \\
\hline Defensive & Defensive & $6.19(1.68)$ \\
\hline General tone $^{a}$ & General tone & $4.90(0.94)$ \\
\hline Activity level $^{\mathrm{a}}$ & Lability skin color ${ }^{\mathrm{a}}$ & $4.60(0.93)$ \\
\hline Regulation of state $(\alpha=.68)$ & Regulation of state $(\alpha=.68)$ & \\
\hline Hand-to-mouth & Hand-to-mouth & $4.49(2.65)$ \\
\hline Self-quieting & Self-quieting & $4.92(2.82)$ \\
\hline Cuddliness & Cuddliness & $6.72(1.42)$ \\
\hline Autonomic stability ( $\alpha=.47$ ) & Autonomic stability $(\alpha=.54)$ & \\
\hline Tremulousness ${ }^{\mathrm{a}}$ & Tremulousness & $2.26(1.95)$ \\
\hline Startles ${ }^{a}$ & Startles & $2.56(1.33)$ \\
\hline Labil. skin color $^{\mathrm{a}}$ & Activity level & $4.84(0.76)$ \\
\hline
\end{tabular}

\footnotetext{
a Recoded items.
} 
Table 5

Correlation between NBAS sub-scales.

\begin{tabular}{|c|c|c|c|c|c|c|c|}
\hline & Habituation & Orientation & Range state ${ }^{a}$ & Motor & Regulation state & Autonomic stability ${ }^{\mathrm{b}}$ & Reflexes $^{\mathrm{b}}$ \\
\hline Habituation & - & & & & & & \\
\hline Orientation & -.17 & - & & & & & \\
\hline Range state ${ }^{a}$ & -.02 & $-.40^{* *}$ & - & & & & \\
\hline Motor & -.09 & $.61^{* *}$ & $-.25^{* *}$ & - & & & \\
\hline Regulation state & .17 & -.08 & $-.23^{* *}$ & $-.18^{*}$ & - & & \\
\hline Autonomic stability ${ }^{\mathrm{b}}$ & .05 & $-.28^{* *}$ & $.19^{*}$ & $-.24^{* *}$ & $.27^{* *}$ & - & \\
\hline Reflexes $^{b}$ & $-.27^{* *}$ & $.25^{* *}$ & -.13 & $.19^{*}$ & $-.25^{* *}$ & $-.25^{* *}$ & - \\
\hline NBAS total score & $.42^{* * *}$ & $.77^{* * *}$ & $-.32^{* * *}$ & $.48^{* * *}$ & $.33^{* * *}$ & $-.16^{* *}$ & $-.19^{* *}$ \\
\hline
\end{tabular}

a Medium score is better performance.

b Higher score is worse performance.

* $p<.01$.

** $p<.05$.

*** $p<.001$

\subsection{Correlation analyses}

Table 5 shows the correlation matrix between components and components/total. Some components are significantly inter-correlated and all components are significantly correlated with the NBAS total score.

\subsection{Construct validity}

Theoretically, we should expect that older infants exhibit better neurobehavioral performance than younger infants. MANOVAs for NBAS component scores indicated a significant effect of age ( 1 week vs 7 weeks) (Wilks' Lambda $=3.45$, $\left.F_{(1,146)}=44.647, p=.000\right)$. Univariate $F$-test shows that 7-week-old infants had significantly better scores on orientation, range of state, motor, regulation of state and autonomic stability factors and worse scores on habituation compared to 1 -week-old infants. No significant differences were found regarding reflexes. As expected, the overall neurobehavioral performance of 7-week-old infants was significantly better compared to 1-week-old infants' neurobehavioral performance (see Table 6).

\section{Discussion}

The principal component analyses reveal a four component structure that explained 55.13\% of the total variance: (1) range of state, composed of four items; (2) motor, composed of five items; (3) regulation of state, composed of four items and (4) autonomic stability, composed of three items. Comparing the extracted component structure with NBAS Lester factors, several similarities were found: (1) range of state component are composed of the same items, (2) regulation of state component are composed of the same items, (3) motor component is composed of four of the items in the Lester motor factor (pull-to-sit, motor maturity, defensive and general tone), (4) autonomic stability is composed of two of the items in the Lester autonomic stability factor (tremulousness and startles). Dissimilarities were also found: (1) the item activity level loads on the autonomic stability component, and (2) lability of skin color loads on the motor component. These dissimilarities across studies might be due to divergent sample characteristics or statistical procedures.

The range of state component seems to be the most consistent across studies since the four items load together in other studies (e.g. Jacobson et al., 1984; Kaye, 1978; Osofsky \& O'Connell, 1977). The other components differ across studies, although similarities are noted with our study: tremulousness and startles load together (Jacobson et al., 1984; Kaye, 1978;

\section{Table 6}

Univariate $F$-test for NBAS factor scores and $t$-test for NBAS total score.

\begin{tabular}{|c|c|c|c|c|}
\hline & 1-week-old mean (SD) & 7 weeks-old mean (SD) & $F$ & $p$ \\
\hline Habituation & $6.85(1.74)$ & $5.43(1.37)$ & 2.075 & .042 \\
\hline Orientation & $6.17(1.40)$ & $8.17(1.10)$ & 79.544 & .000 \\
\hline Range state $^{a}$ & $4.12(1.20)$ & $2.96(1.40)$ & 28.238 & .000 \\
\hline Motor & $5.36(0.74)$ & $6.59(0.57)$ & 107.982 & .000 \\
\hline Regulation state $^{\mathrm{b}}$ & $6.14(1.39)$ & $4.41(1.56)$ & 48.216 & .000 \\
\hline Autonomic stability ${ }^{\mathrm{b}}$ & $3.59(1.16)$ & $2.72(0.58)$ & 25.693 & .000 \\
\hline \multirow[t]{2}{*}{ Reflexes } & $1.43(1.78)$ & $1.50(1.33)$ & .067 & .797 \\
\hline & 1-week-old mean (SD) & 7 weeks-old mean (SD) & $t$ & $p$ \\
\hline NBAS total score & $5.74(0.61)$ & $6.00(0.60)$ & 2.613 & .010 \\
\hline
\end{tabular}

a Medium score is better performance.

b Higher score is worse performance. 
Moragas et al., 2007), general tone, motor maturity and pull-to-sit load in the same factor as well as consolability, self-quieting and hand-to-mouth (Kaye, 1978).

The habituation, orientation and range of state factors have high internal consistency. High internal consistency was also found in other studies in the habituation (Moragas et al., 2007) and orientation factors (Jacobson et al., 1984). Regulation state, motor and autonomic stability Cronbach's alpha are under the recommended .70 (Field, 2005). In other studies, Cronbach's alpha lower than .70 were also reported (Jacobson et al., 1984) which might be due to the low number of items that compose the NBAS factors. Moragas et al. (2007) found values over .70 in four of the five NBAS factors. However, the factors in their study are composed of a higher number of items. The extracted component structure provided by this study is quite similar to the Lester factor structure, when compared to others (e.g., Jacobson et al., 1984; Kaye, 1978; Osofsky \& O'Connell, 1977), strengthening the concept developed by Lester et al. (1982).

Overall, the NBAS indicates a reliable documentation of the newborn behavioral organization. The identification of components in the NBAS scale that mirror specific aspects of the developmental tasks of the newborn is essential for the assessment of their capacities, strengths, adaptative responses and possible vulnerabilities across different developmental areas. This is relevant not only for research but also for clinical practice. For research, the discrimination of specific aspects of neurobehavior is important because it enables the researcher to look at the impact of different circumstances (such as maternal factors of nutrition, psychopathology or substance consumption) on autonomic, motor, state and social-attention systems. It also allows looking at the association of the newborn's different system status with the infant development, and understanding the mechanisms underlying those relations. For clinicians, it is important for understanding newborn's system characteristics and behavioral responses and sharing with the parents the possible needs for extra care giving support in specific developmental areas.

This study has several limitations including the sample size and lack of other infant developmental measures; a large sample size would have increased the power of the results and other infant developmental measures would allow examining concurrent and predictive validity. Future research should analyze the psychometric properties of the NBAS in medical samples (e.g. premature infants, infants exposed to drugs prenatally) and establish a clinical cut-off for the NBAS.

\section{Acknowledgements}

We would like to thank to the participants in this study and to the Primary Health Center of Espinho and Santa Maria da Feira and Maternity Júlio Dinis. We would also like to thank Filomena Louro for writing assistance and English language corrections. Financed by the Operational Program Science and Innovation 2010 (POCI 2010) of the III Community Support and co-financed by the European Community Found FEDER (POCI/SAU-ESP/56397/2004; Anxiety and depression in women and men in the transition to parenthood: Effects on fetal and neonatal development and behaviour) and under a FCT PhD Grant (SFRH/BD/18249/2004).

\section{References}

Als, H. (1978). Assessing an assessment: Conceptual considerations, methodological issues, and a perspective on the future of the neonatal behavioral assessment scale. Monographs of the Society for Research in Child Development, 43(5-6, no. 177), 14-28.

Azuma, S. D., Malee, K. M., Kavanagh, J. A., \& Deddish, R. B. (1991). Confirmatory Factor Analysis with preterm NBAS data: A comparison of four data reduction Models. Infant Behavior and Development, 14, 209-225.

Boatella-Costa, E., Moragas, C. C., Mussons, F. B., Deu, A. F., \& Zurita, M. L. (2007). Behavioral gender differences in the neonatal period according to the Brazelton scale. Early Human Development, 83, 91-97.

Brazelton, T. B. (1973). Neonatal Behavioral Assessment Scale. Philadelphia: Lippincott.

Brazelton, T. B., \& Nugent, J. K. (1995). Neonatal Behavioral Assessment Scale. Cambridge: Cambridge, University Press.

Field, A. (2005). Discovering statistics using SPSS. London: SAGE Publications.

Field, T., Diego, M., Hernandez-Reif, M., Schanberg, S., \& Kuhn, C. (2002). Relative right versus left frontal EEG in neonates. Developmental Psychobiology, 41(2), 147-155.

Jacobson, J. L., Fein, G. G., Jacobson, S. W., \& Schwartz, P. M. (1984). Factors and clusters for the Brazelton Scale: An investigation of the dimensions of neonatal behavior. Developmental Psychology, 20, 339-353.

Kaiser, H. F. (1960). The application of electronic computers to factor analysis. Educational and Psychological Measurement, 20, 141-151.

Kaiser, H. F. (1974). An index of factorial simplicity. Psychometrika, 39, 31-36.

Kaye, K. (1978). Discriminating among normal infants by multivariate analysis of brazelton scores: Lumping and smoothing. Monographs of the Society for Research in Child Development, 43(5-6, no. 177), 60-80.

Lester, B. M., Als, H., \& Brazelton, T. B. (1982). Regional obstetric anesthesia and newborn behavior: A reanalysis toward synergistic effects. Child Development, $53,687-692$

Loo, K. K., Ohgi, S., Zhu, H., Howard, J., \& Chen, L. (2005). Cross-cultural comparison of the neurobehavioral characteristics of Chinese and Japanese neonates. Pediatrics International, 47, 446-451.

McCollam, K. M., Embretson, S. E., Horowitz, F. D., \& Mitchell, D. W. (1996). Scoring the NBAS: To recode or not to recode. Infant Behavior E Development, 19, 63-69.

McCollam, K. M., Embretson, S. E., Mitchell, D. W., \& Horowitz, F. D. (1997). Using confirmatory factor analysis to identify newborn behavior structure with the NBAS. Infant Behavior \& Development, 20(2), 123-131.

Moragas, C. C., Deu, A. F., Mussons, F. B., Boatella-Costa, E., \& Zurita, M. L. (2007). Evaluación psicométrica de la Escala de Brazelton en una muestra de recién nacidos espanoles. Psicothema, 19(1), 140-149.

Myers, B. J., Dawson, K. S., Britt, G. C., Lodder, D. E., Meloy, L. D., Saunders, M. K., et al. (2003). Prenatal cocaine exposure and infant performance on the Brazelton Neonatal Behavioral Assessment Scale. Substance Use E' Misuse, 38, 2065-2096.

Nunnally, J., \& Bernstein, I. (1994). Psychometric theory (3rd ed.). New York: MacGraw-Hill.

Ohgi, S., Arisawa, K., Takahashi, T., Kusumoto, T., Goto, Y., Akiyama, T., et al. (2003). Neonatal behavioral assessment scale as a predictor of later developmental disabilities of low birth-weight and/or premature infants. Brain $\mathcal{E}$ Development, 25, 313-321. 
Ohgi, S., Fukuda, M., Akiyama, T., \& Gima, H. (2004). Effect of an early intervention programme on low birthweight infants with cerebral injuries. Journal of Paediatric Child Health, 40(12), 689-695.

Osofsky, J. D., \& O'Connell, E. J. (1977). Patterning of newborn behavior in an urban population. Child Development, 48(2), 532-536.

Sostek, A., Davitt, M. K., Renzi, J., Jr., Born, W. S., \& Kiely, S. C. (1982). Factor analysis of behavioral assessments of preterm neonates. In L. P. Lipsitt, \& T. M. Field (Eds.), Infant behavior and development: Perinatal risk and newborn behavior. Norwood, NJ: Ablex.

Strauss, M. E., \& Rourke, D. I. (1978). A multivariate analysis of the neonatal behavioral assessment scale in several samples. Monographs of the Society for Research in Child Development, 43(5-6, no. 177), 81-91. 